Accretion Phenomena and Related Outflows, IAU Colloquium 163

ASP Conference Series, Vol. 121, 1997

D.T. Wickramasinghe, L. Ferrario, and G.V. Bicknell, eds.

\title{
Water Maser Emission from Dusty Clouds in AGNs
}

\author{
John F. Kartje and Arieh Königl \\ Dept. of Astronomy \&3 Astrophysics, Univ. of Chicago, 5640 S. Ellis \\ Ave., Chicago, IL 60637, U.S.A. \\ Moshe Elitzur \\ Dept. of Physics 63 Astronomy, Univ. of Kentucky, Lexington, $K Y$ \\ 40506, U.S.A.
}

\begin{abstract}
A natural site for water maser emission in AGNs is provided by dusty gas with properties characteristic of broad line region (BLR) clouds. Radiation shielding by dust in the clouds is critical for allowing molecular gas to exist $\leq 1$ pc from the central engine. Thus, the innermost radius at which such masers appear should correspond to the grain sublimation radius $r_{s u b}$. We suggest a dynamical model in which the masing clouds are embedded within a magnetized accretion disk wind.
\end{abstract}

\section{Masing in Dusty BLR Clouds}

We employed the photoionization code CLOUDY (Ferland 1996) to compute the run of temperature $T$ and fractional water abundance (with respect to hydrogen) $x\left(\mathrm{H}_{2} \mathrm{O}\right)$ as a function of depth into uniform-density clouds irradiated by an external continuum source. The cloud density $n=5 \times 10^{9} \mathrm{~cm}^{-3}$ and radius $R=10^{14} \mathrm{~cm}$ were chosen to be representative of values deduced from BLR emission line studies (e.g., Baldwin et al. 1995), while the intrinsic spectrum inferred for NGC 1068 (Pier et al. 1994) was used for the incident flux.

We have confirmed that dust plays a seminal role in the formation of molecular gas within the clouds. Figure 1 presents the temperature profiles for clouds, both with and without dust, located $0.4 \mathrm{pc}$ from the radiation source. Clouds at distances $r_{s u b} \leq r \leq 1$ pc typically have hot, atomic outer layers and cool molecular interiors with significant water abundances (i.e, $x\left(\mathrm{H}_{2} \mathrm{O}\right) \geq 10^{-5}$ ), while dust-free clouds remain hot and atomic throughout their interiors. As long as $T \geq 250 \mathrm{~K}, \mathrm{H}_{2} \mathrm{O}$ masers can be collisionally pumped. We find that dusty BLR clouds serve as efficient masers out to distances $\sim 1 \mathrm{pc}$. This range compares closely with the observed position of the masing gas in the Seyfert 2 galaxy NGC 1068 (Greenhill et al. 1996).

\section{Masing Clouds in Disk Winds}

We propose that the dynamics of masing, dusty clouds could be governed by centrifugally driven magnetized accretion disk winds. Königl \& Kartje (1994; 

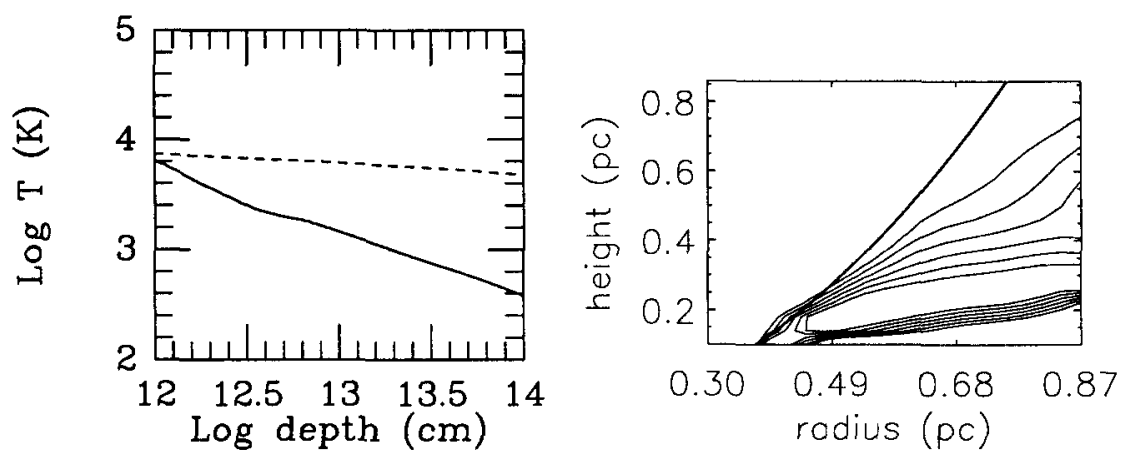

Figure 1. Left Panel: $T$ vs. depth for a dusty cloud (solid curve) and dust-free cloud (dashed curve). Right Panel: Contours of maser brightness temperature for dusty clouds embedded in a magnetized disk wind; the relative increase in $T_{b}$ from the outermost to the innermost contour is a factor of 2 . The heavy curve indicates the position of the innermost wind stream line along which dust can survive in the radiation field.

KK94) demonstrated that such winds can uplift gas and dust from the surface of a dense molecular disk and could serve as the "obscuring torus" considered to be a common component of Seyfert galaxies. We expect that clouds lifted from the disk would tend to follow the wind stream lines and would be confined as the BLR emission line clouds are, either by external gas pressure or by the wind magnetic field. Figure 1 presents the contours of maser brightness temperature $T_{b}$ for clouds in a representative wind model (the wind parameters correspond to model "A1" in KK94). The tendency for the $T_{b}$ contours to rise from the midplane of the disk is similar to that seen in VLBI observations of masing gas in NGC 1068 (Greenhill et al. 1996). We have also confirmed that the cloud velocities along the wind streamlines display a non-Keplerian profile similar to that observed in this source. Full details of this model are presented in Kartje, Königl, \& Elitzur (1996).

\section{References}

Baldwin, J., Ferland, G., Korista, K., \& Verner, D. 1995, ApJ, 455, L119

Ferland, G. J., 1996, Hazy, a Brief Introduction to Cloudy, Univ. of Kentucky Dept. of Physics \& Astronomy Internal Report

Greenhill, L. J., Gwinn, C. R., Antonucci, R., \& Barvainis, R. 1996, ApJ, submitted

Kartje, J. F., Königl, A., \& Elitzur, M. 1996, ApJ, in preparation

Königl, A., \& Kartje, J. F. 1994, ApJ, 434, 446

Pier, E. A., Antonucci, R. R. J., Hurt, T., Kriss, G., \& Krolik, J. 1994, ApJ, 428,124 